\title{
SEGREGAÇÃO ESCOLAR NA CIDADE DO RIO DE JANEIRO: ANÁLISE DA MOVIMENTAÇÃO DE ESTUDANTES
}

TIAGO LISBOA BARTHOLO

\section{RESUMO}

0 artigo analisa o fenômeno das transferências de alunos entre escolas municipais da cidade do Rio de Janeiro e suas possíveis relações com processos de segregação escolar. É utilizado um indicador para medir a segregação escolar - o Segregation Index -, em desenhos longitudinais para todas as transições do ensino fundamental. Os dados foram fornecidos pela Secretaria Municipal de Educação e o contemplam todos os alunos da rede entre 2006 e 2011. Três características dos alunos foram consideradas: cor/raça; educação dos pais; $e$ condição de pobreza. Os resultados sugerem que: o impacto do remanejamento de alunos entre o primeiro e o segundo segmentos é desagregador, ou seja, as escolas se tornam mais misturadas em sua composição social; as transferências ao longo do segundo segmento apresentam um padrão definido e sugerem um processo constante de seleção do alunado. 


\section{RESUMEN}

El artículo analiza el fenómeno de las transferencias de alumnos entre escuelas municipales de la ciudad de Rio de Janeiro y sus posibles relaciones con procesos de segregación escolar. Se utiliza un indicador para medir la segregación escolar - el Segregation Index -, en diseños longitudinales para todas las transiciones de la educación fundamental. Los datos fueron suministrados por la Secretaría Municipal de Educación y contemplan todos los alumnos de la red entre el 2006 y el 2011. Se consideraron tres características de los alumnos: color/raza; educación de los padres; $y$ condición de pobreza. Los resultados sugieren que el impacto del traslado de alumnos entre el primero y el segundo segmentos es disgregador, es decir, las escuelas adquieren más mezcla en su composición social; las transferencias a lo largo del segundo segmento presentan un estándar definido y sugieren un proceso constante de selección del alumnado.

PALABRAS CLAVE SEGREGACIÓN ESCOLAR - MOVIMIENTO DE ESTUDIANTES • ESTUDIO LONGITUDINAL • INDICADORES.

\section{ABSTRACT}

This article analyzes the phenomenon of relocating students among the public schools in the city of Rio de Janeiro, and the possible relationships with school segregation processes. An indicator - the Segregation Index - was used to measure school segregation in longitudinal designsof all transitions of elementary school. Data were supplied by the Secretary of Municipal Education, and include all students in the system between 2006 and 2011. Three characteristics of students were considered: color/race, education of the parents, and poverty level. Results suggest that the impact of student relocation between the basic and secondary levels is disruptive; that is, schools become more mixed in their social composition; the relocations at the secondary level present a definite pattern and suggest a constant process of student selection.

KEY WORDS SCHOOL SEGREGATION - RELOCATION OF STUDENTS • LONGITUDINAL STUDY • INDICATORS. 


\section{INTRODUÇÃO}

$\mathrm{O}$ artigo analisa o fenômeno das transferências de alunos entre escolas públicas da rede municipal do Rio de Janeiro e sua relação com processos de segregação escolar. A hipótese inicial sugere que as frequentes trocas de alunos entre escolas apresentam um padrão específico, que associa o perfil do aluno com a composição social das escolas. O tema não é novo e já foi investigado no Rio de Janeiro e em outras cidades do Brasil (ALVES; SOARES, 2008; BRUEL; BARTHOLO, 2012; COSTA et al., 2014). Há, no entanto, três aspectos do artigo que são originais: o uso de indicadores de segregação escolar para descrever a distribuição de alunos na rede; o emprego de dados completos sobre a população de alunos na rede pública municipal do Rio de Janeiro; e a análise de todas as transições ao longo do ensino fundamental.

Estudos anteriores sobre a rede municipal do Rio de Janeiro analisaram a transição entre o primeiro e o segundo segmentos, chamada de remanejamento, a qual possui uma característica especial: ser obrigatória para a maioria dos alunos. Aproximadamente $90 \%$ dos alunos são obrigados a mudar 
de escola, uma vez que não há oferta da série subsequente no mesmo estabelecimento. O presente artigo analisa todas as transições ao longo do ensino fundamental e propõe novas interpretações para estudos previamente publicados sobre essa temática.

As desigualdades educacionais podem se manifestar de diferentes formas, sendo que pesquisadores adotam diferentes objetos de estudo: distribuição de recursos; a chance de um aluno frequentar uma escola de alta performance; diferença no desempenho entre alunos de baixo e alto nível socioeconômico; acesso a um currículo mais acadêmico (JENKS, 1972). Esse trabalho foca nas possíveis causas da segregação escolar, ou, de forma mais específica, nos impactos não intencionais na segregação escolar provocados por práticas da burocracia educacional. Estudos anteriores identificaram que a ausência de uma legislação clara que regule as transferências pode facilitar a troca de alunos entre escolas, ampliando as desigualdades de oportunidades educacionais (BRUEL; BARTHOLO, 2012).

O tema da segregação está diretamente ligado à temática da qualidade e equidade dos sistemas educacionais e, de forma mais ampla, à justiça social. Nas sociedades atuais, as escolas tornaram-se uma instituição central que deveria prover, pelo menos na teoria, oportunidades iguais para todos, viabilizando mobilidade social e melhores condições de vida.

Evidências de diferentes países, incluindo o Brasil, sugerem que concentrar alunos com características específicas (aqui chamados de alunos em desvantagem potencial) em determinadas escolas pode influenciar a forma como eles são tratados, a qualidade do ensino e a aspiração para os níveis subsequentes de educação. Há ainda indicações de que sistemas educacionais mais segregados ou com políticas que intencionalmente agrupam alunos em desvantagem potencial possuem maior associação entre os níveis de aprendizagem e o perfil socioeconômico dos alunos, ou seja, alunos com perfil socioeconômico mais desfavorável apresentam desempenho inferior (HAAHR et al., 2005; EUROPEAN GROUP..., 2005; JENKS et al., 1972; ROSENTHAL; JACOBSON, 1968; BRITO; COSTA, 2010; BARTHOLO, 2014). 
A seguir, apresenta-se um debate sobre o que é desvantagem em educação. Posteriormente, são expostos os dados sobre a rede municipal do Rio de Janeiro e sobre estudos anteriores que analisaram a transição entre o primeiro e o segundo segmentos do ensino fundamental. É realizada, ainda, uma descrição sobre o indicador de segregação e os desenhos do estudo considerando todas as transições escolares. Finalmente, discutem-se os principais achados do estudo e são colocadas propostas para algumas questões levantadas ao longo do artigo.

\section{O QUE É DESVANTAGEM EM EDUCAÇÃO?}

Essa seção apresenta as variáveis-chave utilizadas no estudo para medir segregação escolar e discute de forma mais aprofundada o que é desvantagem em educação. Há um consenso por parte da comunidade científica de que as oportunidades educacionais e os resultados educacionais são estratificados pelo perfil socioeconômico dos alunos. A força da associação entre aprendizagem e nível socioeconômico varia entre países, mas a correlação está presente em todos os sistemas educacionais em que há dados disponíveis.

Estudos internacionais mostram que os níveis de aprendizagem são altamente correlacionados com algumas características-chave dos alunos, tais como cor, estrutura familiar e ocupação, anos de escolarização e renda dos pais. Para os anos iniciais do ensino fundamental, os principais fatores que impactam a aprendizagem são educação dos pais, pobreza, desemprego e mães adolescentes. Todos os estudos reforçam que alunos em desvantagem potencial têm maior chance de apresentar baixo rendimento escolar. Essas são evidências robustas de um padrão bastante claro, que associa desigualdades sociais (por exemplo, ser pobre) com baixo desempenho escolar (GORARD; SEE, 2013).

Esse é talvez o maior desafio para profissionais que atuam no campo da educação. Como reduzir de forma eficiente a associação entre o nível socioeconômico dos alunos e os níveis de aprendizagem? Compreender como funciona o "gradiente pobreza" é particularmente importante para 
os gestores públicos que devem planejar intervenções eficazes, cujo objetivo é ajudar os educadores e as famílias dos alunos. Como superar a desvantagem educacional inicial é claramente uma questão causal, que demanda um esforço por parte dos pesquisadores na busca de desenhos avaliativos mais robustos. Isso já vem sendo feito em países como Estados Unidos, Inglaterra e Holanda, de forma mais intensa a partir da década de 1990, com o uso cada vez mais frequente de experimentos randomizados ou estudos longitudinais, que apresentam melhor qualidade na inferência causal (FITZ-GIBBON, 1996).

Há uma ampla gama de explicações sobre como a desvantagem inicial pode afetar o rendimento dos alunos na escola. É razoável presumir que as oportunidades educacionais são fortemente afetadas por padrões de segregação residencial. Bolsões de pobreza dentro das grandes cidades podem produzir isolamento social (WILSON, 1987) e ter um impacto na qualidade das escolas - por exemplo, na infraestrutura e no recrutamento de professores. A concentração de alunos em desvantagem potencial em determinadas áreas ou escolas também afeta o acesso às informações relevantes, que podem impactar oportunidades futuras, retirar lideranças positivas, dificultar o recrutamento de professores mais qualificados, diminuir os níveis de aspiração e motivação para o trabalho escolar e prejudicar o sentimento de pertencimento que os alunos têm sobre sua inserção na sociedade. Todos esses elementos podem impactar de forma direta ou indireta o aprendizado dos alunos.

Qualquer tentativa de superar a desvantagem educacional não deve ignorar a temática sobre a alocação dos alunos nas escolas. A concentração de alunos com características semelhantes é um fenômeno relevante e universal. Estudos internacionais em grande escala (por exemplo, Pisa, TIMSS e PIRLS) mostram a viabilidade de sistemas educacionais combinarem alta qualidade (elevada proporção de alunos com níveis adequados de aprendizagem) com alta equidade (baixo desvio-padrão dos níveis de aprendizagem) (EUROPEAN GROUP..., 2005).

Políticas educacionais precisam ser encaradas como causas maleáveis da segregação escolar, com possíveis impactos 
nos níveis gerais de aprendizagem, devendo ser uma prioridade para os gestores públicos que desejam construir sistemas educacionais mais eficazes e justos. Cabe ressaltar que mudanças nas regras de admissão ou regulação das transferências de alunos não apresentam, em geral, custo financeiro adicional para os gestores (no máximo, um custo político). Trata-se de uma decisão política que tem o potencial de afetar diretamente as oportunidades educacionais de alunos e suas chances futuras de mobilidade social. O presente estudo objetiva contribuir para o debate sobre o impacto das políticas educacionais no processo de alocação dos alunos.

\section{SEGREGAÇÃo ESCOLAR NA REDE PÚBLICA MUNICIPAL DO RIO DE JANEIRO: EVIDÊNCIAS DE ESTUDOS ANTERIORES}

A rede pública municipal do Rio de Janeiro está entre as maiores redes de ensino da América Latina, com aproximadamente 1.300 escolas e 750 mil alunos divididos em pré-escola e ensino fundamental. É importante reforçar que, na cidade do Rio de Janeiro, cerca de $26 \%$ das matrículas no ensino fundamental pertencem à rede privada de ensino, parcela essa, portanto, desprezada pelo presente artigo. Estudos futuros devem fazer um esforço para incorporar dados do Inep sobre as escolas privadas com intuito de replicar os modelos expostos e confirmar os resultados preliminares encontrados.

Na rede pública municipal do Rio de Janeiro não há restrições formais para a matrícula relacionadas à localização da moradia dos alunos. Mesmo na ausência de políticas claras que incentivem a escolha de estabelecimentos escolares, é possível afirmar que as famílias optam por diferentes escolas a partir de critérios específicos (COSTA; PRADO; ROSISTOLATO, 2013).

Estudos anteriores analisaram os procedimentos para a matrícula na rede municipal do Rio de Janeiro e identificaram o que foi denominado "brechas na legislação". A ausência de especificações claras sobre determinados procedimentos no momento da matrícula inicial ou transferência permitia 
que diretores ou outros agentes da burocracia educacional selecionassem alunos em diferentes etapas do processo de escolarização.

Em 2011, as regras de admissão inicial de alunos e, posteriormente, os pedidos de mudança de escola ao longo do primeiro e segundo segmentos (aqui ficam excluídas as transferências no remanejamento) foram alterados. Um novo sistema informatizado e mais impessoal passou a reger o processo de admissão dos alunos. As famílias preenchem uma lista com até cinco opções de escolas e um software faz a alocação dos alunos de forma aleatória. O novo sistema de alocação de alunos tem o potencial para mudar os padrões de transferências de alunos entre escolas, mas não será abordado neste artigo. Qualquer análise futura sobre os impactos das novas regras de admissão deverá comparar os padrões anteriores de trocas de alunos (descritos neste estudo) com os novos padrões que possivelmente se iniciaram em 2012.

Bruel e Bartholo (2012) e Costa et al. (2014), utilizando as mesmas bases de dados deste artigo, analisaram as transferências de alunos no remanejamento (2008 e 2009) em um número limitado de escolas. Os autores selecionaram as mesmas variáveis para estimar a chance de acesso a escolas de alta performance na rede pública. As conclusões preliminares dos estudos são semelhantes e sugerem que o efeito geral da realocação de alunos no remanejamento torna as escolas mais homogêneas, com potencial para aumentar a segregação escolar. Tal efeito é atribuído à ação deliberada dos diretores de escolas na seleção de alunos com determinadas características. Segundo os autores, o melhor conceito para explicar o fenômeno seria "ecologia do mercado escolar", cunhado por Yair (1996) - um sistema de colaboração entre escolas.

Ambos os estudos apresentam três limitações metodológicas. A primeira está relacionada ao "ponto de corte" para a divisão das escolas em dois grupos (alta e baixa performance). Trata-se de uma divisão arbitrária, em geral considerando o quartil superior nas avaliações externas padronizadas (Prova Brasil ou Prova Rio) como escolas de alta performance. Uma questão não respondida pelos estudos é: mudando os 
critérios para a divisão das escolas (por exemplo, dividindo em dois grupos iguais), os resultados seriam consistentes?

A segunda limitação é o critério utilizado para selecionar as escolas que seriam analisadas. Amostra intencional, com um número pequeno de escolas, impede a extrapolação dos resultados para o restante da rede e embute o risco de viés de seleção amostral. Por fim, no que se refere à terceira limitação, é importante ressaltar que um modelo que objetiva medir o impacto do remanejamento na segregação escolar deve comparar medidas sobre a segregação escolar antes e após a transição. Os estudos anteriores apresentam modelos que permitem observar o fenômeno da seleção de alunos, sendo que os coeficientes das regressões logísticas ali encontrados não devem ser interpretados como indicativo de um aumento dos níveis de segregação (BARTHOLO, 2014).

Os modelos apresentados neste artigo buscam eliminar todas as limitações supracitadas. Trabalhar com dados para todas as escolas da rede elimina o risco de viés de seleção amostral e permite a descrição dos resultados de forma direta e objetiva. Outro incremento é o uso de indicadores de segregação, que permitem abandonar o modelo anterior que separava as escolas em grupos arbitrários. Por fim, os modelos longitudinais possibilitam comparar medidas de segregação antes e após o remanejamento, descrevendo de forma objetiva as mudanças nos níveis de segregação.

\section{DESENHO DO ESTUDO E INDICADORES DE SEGREGAÇÃO ESCOLAR}

Segregação escolar é compreendida, neste estudo, como uma distribuição desigual de alunos com uma característica compartilhada (aqui denominados "alunos em desvantagem potencial”) e será medida por um indicador chamado Segregation Index (GS).

O GS apresenta uma interpretação simples e direta, que permite a compreensão instantânea do fenômeno: indica a proporção de alunos em desvantagem potencial que deveriam mudar de escola para obter uma distribuição equânime - 0\% 
de segregação. Esse indicador pode ser descrito a partir da seguinte fórmula:

$$
\mathrm{GS}=0.5 *\left\{\sum|\mathrm{Fi} / \mathrm{F}-\mathrm{Ti} / \mathrm{T}|\right\},
$$

onde:

Fi é o número de alunos em desvantagem potencial na escola $i$, em que $i$ varia de 1 até o número total de escolas na rede; F corresponde ao número total de alunos em desvantagem potencial nas escolas públicas do Rio de Janeiro; Ti é o número de alunos na escola $i$, em que $i$ varia de 1 até o total de escolas na rede; e $T$ refere-se ao total de alunos nas escolas públicas do Rio de Janeiro (GORARD; TAYLOR; FITZ, 2003).

$\mathrm{O}$ índice sumariza um valor único, indicando o quão desigual é a distribuição de alunos em desvantagem potencial em um conjunto de escolas. Há muitos índices disponíveis no "mercado" e é relevante compreender a adequação (pontos fortes e fracos) de cada indicador antes de realizar sua esco1ha. Gorard (2009) destaca quatro características desejáveis que os indicadores devem apresentar, independentemente do campo de atuação:

1) invariante na organização (organisationally invariant), de forma que, quando uma escola é dividida em duas, ou se duas escolas se tornam uma, mantendo a mesma proporção de alunos em desvantagem potencial em cada unidade, o valor do indicador deve permanecer o mesmo; 2) invariante no tamanho ou escala (size or scale invariant), de forma que, multiplicando por uma constante em todas as escolas o número de alunos em desvantagem e os demais, o valor do indicador permanece o mesmo; 3) invariante na composição (compositionally invariant), de forma que, multiplicando por uma constante em todas as escolas o número de alunos em desvantagem, o valor do indicador permanece o mesmo; 4) afetado pelas transferências, de forma que, se um aluno em desvantagem muda de uma escola com alta proporção de alunos em desvantagem para outra com menor proporção, o indicador apresenta um declínio nos níveis de segregação. (GORARD, 2009, p. 644, tradução nossa) 
O Segregation Index (GS) apresenta um resultado semelhante ao Índice de Dissimilaridade (D) (DUNCAN; DUNCAN, 1955), com a vantagem de possuir uma interpretação mais clara e direta. Uma das principais preocupações de pesquisadores que utilizam índices de segregação em modelos longitudinais é ter a certeza que os valores calculados não irão se alterar apenas com a simples mudança na proporção total de alunos em desvantagem potencial em cada região. Como é muito provável que ocorram pequenas flutuações na proporção de alunos em desvantagem ao longo dos anos, análises longitudinais exigem indicadores que sejam compositionally invariant.

Gorard e Taylor (2002) fazem uma distinção entre D e GS, destacando que o primeiro é "pouco invariante na composição" (weakly composition invariant), enquanto o segundo é "muito invariante na composição" (strongly composition invariant). GS é o indicador selecionado nesse artigo. No entanto, análises anteriores utilizando os mesmos dados da Secretaria Municipal de Educação compararam os resultados com ambos os indicadores e reforçaram que medir segregação escolar empregando D ou GS é tão diferente quanto medir a temperatura de um ambiente pela escala Celsius ou Fahrenheit (BARTHOLO, 2013; 2014).

$\mathrm{O}$ artigo usa dados secundários disponibilizados pela Secretaria Municipal de Educação do Rio de Janeiro (SME-RJ). Trata-se de uma base de dados longitudinal com informações no nível aluno. Uma descrição detalhada do trabalho de "limpeza" dos bancos e a caracterização dos dados faltantes (missing) podem ser encontradas em Bartholo (2013; 2014). Em linhas gerais, as informações permitem acompanhar a trajetória escolar dos alunos ao longo dos anos, suas mudanças de escola, turno e turma e traçar um perfil dos alunos.

Para este artigo, foram selecionadas três variáveis para descrever os alunos em desvantagem potencial: educação dos pais; condição de pobreza; e cor/raça. Essas características têm sido utilizadas em estudos internacionais e nacionais sobre o tema da segregação escolar e apresentam associação com o desempenho escolar. 
Educação dos pais é possivelmente o melhor preditor da trajetória escolar de uma criança, com exceção de uma medida direta de proficiência. Mesmo em países com altas taxas de mobilidade social, como a Dinamarca, a ocupação e o nível de escolaridade dos pais são os melhores preditores do sucesso escolar de uma criança. Controlando todas as variáveis conhecidas que podem afetar o desempenho escolar (incluindo medidas iniciais de proficiência), crianças com pais mais educados têm maiores chances de sucesso na escola (GORARD; SEE, 2013).

Nas bases da SME-RJ, educação dos pais é uma variável ordinal de cinco pontos: analfabeto; não concluiu o ensino fundamental; concluiu o ensino fundamental; ensino médio completo; e ensino superior completo. Para elaboração dos indicadores de segregação, a variável foi reconstruída (resumida), criando-se dois grupos distintos de alunos em desvantagem potencial: pais que não terminaram o ensino fundamental (EducEF); e pais que não terminaram o ensino médio (EducEM).

O efeito da pobreza sobre o desempenho escolar é um dos temas centrais no campo da educação e da justiça social. Superar a desvantagem educacional inicial é, entre outras coisas, diminuir a distância do desempenho escolar entre os que nasceram na pobreza e o resto da população. Reduzir a pobreza em si é uma das principais metas das democracias ocidentais (incluindo organismos internacionais, como a Unesco), sendo que os sistemas educacionais exercem papel importante nesse desafio. Infelizmente, há poucas iniciativas sistemáticas no campo educacional para compreender os mecanismos que levam crianças pobres a apresentarem baixo rendimento.

No caso brasileiro, pobreza é provavelmente a variável mais importante para ser medida e acompanhada por pesquisadores e gestores públicos. Bartholo (2014) demonstrou que essa é a variável que apresenta a maior correlação com as demais características de desvantagem potencial nas escolas públicas do Rio de Janeiro.

Desde 1990, o governo federal, em conjunto com administrações estaduais e municipais, implementou algumas 
1 "O Cadastro Único para Programas Sociais do Governo Federal, instituído pelo Decreto n. 6.135/07, é um instrumento de identificação e caracterização socioeconômica das famílias brasileiras de baixa renda, entendidas prioritariamente como aquelas cuja renda per capita mensal é de até meio salário mínimo. Famílias com renda superior (até três salários mínimos) também podem ser cadastradas, para o planejamento ou implementação de programas sociais específicos. Dessa forma, o número de famílias cadastradas é maior que a quantidade de famílias beneficiadas pelo PBF." Ver: <http://www.mds.gov. $\mathrm{br} /$ falemds/perguntas-frequentes/ bolsa-familia/cadastro-unico/ beneficiario/cadunico-inclusao>.

2 Nossos estudos qualitativos indicam que há falha no protocolo de coleta de dados sobre o perfil dos alunos, em especial para a informação sobre cor. Conversando com diretores

de escolas e pais de alunos, foi possível identificar que, em alguns casos, a informação é obtida a partir de autodeclaração, porém, há relatos de situações nas quais o agente da escola preenche a informação a partir de observação.

3 Ao longo dos anos a nomenclatura dos programas mudou. Neste estudo, estamos especialmente interessados no envio de alunos para os programas de realfabetização (seleciona alunos no terceiro ano do ensino fundamental) e para as classes de progressão (segundo segmento do ensino fundamental). políticas públicas - chamadas de políticas sociais -, visando a diminuir os níveis de pobreza no Brasil. Políticas de transferência de renda foram criadas e aperfeiçoadas ao longo dos anos. Nas bases da SME-RJ, há informação sobre o Número de Inscrição Social (NIS), ${ }^{1}$ que permite identificar famílias em situação de vulnerabilidade social e que, em tese, são elegíveis para receber benefício de algum programa social do Estado. Trata-se de uma proxy de pobreza.

A terceira variável, cor/raça do aluno, tem sido utilizada em diversos estudos no Brasil e em outros países para compreender desigualdades sociais relacionadas não apenas à temática das oportunidades educacionais, mas também ao acesso ao mercado de trabalho, renda, exposição à violência, etc. Essa variável foi construída distinguindo os que se declararam $^{2}$ pretos (aqui caracterizados como alunos em desvantagem potencial) dos demais alunos.

O estudo apresenta três desenhos complementares que analisam o impacto das transferências de alunos na segregação escolar. Uma das formas de captar a movimentação dos alunos ao longo das transições é selecionar uma coorte específica de alunos e calcular o indicador nos anos subsequentes à medida que os alunos progridem na escola. Caso as transferências sejam aleatórias, sem uma associação clara entre o perfil do alunado e a composição social das escolas, é esperado que o índice apresente uma trajetória estável ao longo do tempo. No entanto, caso as transferências tenham um padrão específico, associando perfil dos alunos e composição social das escolas, o índice deverá apresentar um aumento dos níveis gerais de segregação.

Idealmente, o desenho do estudo poderia ser transversal, porém há dois elementos que podem influenciar diretamente os resultados desse modelo: repetência; e envio de alunos para os programas de reforço escolar ${ }^{3}$ ou Educação de Jovens e Adultos (EJA). Em função disso, é apresentado um total de três desenhos complementares que objetivam controlar outras variáveis que podem influenciar o resultado sobre as transferências de alunos na segregação escolar.

O primeiro desenho do estudo é transversal. Duas coortes foram selecionadas para as análises: coorte de 2006 com todos 
os alunos matriculados no $1^{\circ}$ ano do ensino fundamental; e coorte de 2006 com todos os alunos matriculados no $6^{\circ}$ ano do ensino fundamental. Os dados da coorte do $1^{\circ}$ ano foram utilizados para analisar o primeiro segmento do ensino fundamental e a transição entre o primeiro e o segundo segmentos - remanejamento. Com os dados da coorte do $6^{\circ}$ ano, analisaram-se as transferências ao longo do segundo segmento.

O segundo modelo é longitudinal e empregou dados das mesmas coortes. A diferença é que os mesmos alunos foram seguidos ao longo das transições, de forma que novos alunos não foram incorporados na análise e excluíram-se aqueles que foram reprovados. Esse desenho permite observar de forma mais clara o potencial da reprovação e das transferências na segregação escolar.

O último desenho também é longitudinal e utilizou dados das mesmas coortes, porém os alunos reprovados ou enviados para classes de realfabetização (primeiro segmento) ou de progressão ou EJA (segundo segmento) foram mantidos da análise. Esse desenho busca isolar o efeito das transferências de alunos na segregação escolar.

\section{PRIMEIRO SEGMENTO DO ENSINO FUNDAMENTAL}

Esta seção apresenta os dados para todas as transições do primeiro segmento do ensino fundamental. A hipótese inicial afirma que as trocas de alunos entre escolas possuem um padrão específico, que associa o perfil dos alunos à composição social das escolas. As cinco séries iniciais do ensino fundamental na SME-RJ englobam aproximadamente 800 escolas e 320 mil alunos - os valores mudam ligeiramente dependendo do ano analisado. Na coorte estudada, alunos do $1^{\circ}$ ano de 2006 representam 68.926 e há uma perda de casos (attrition rate) de 0,6\% ao final das quatro transições. Esses são alunos para os quais não havia informações sobre a sua trajetória escolar em algum momento entre 2006 e 2010. Para uma descrição detalhada sobre os problemas e limitações das bases da SME-RJ, ver Bartholo (2014).

Uma questão inicial que precisa ser respondida é: qual a proporção exata de alunos que trocam de escolas ao 
longo do primeiro segmento? Trata-se de uma questão-chave, que permite compreender a intensidade do fenômeno. É importante destacar que o único período obrigatório de troca de escolas para a maioria dos alunos é do $5^{\circ}$ para o $6^{\circ}$ ano (remanejamento). As transferências descritas nesta seção são motivadas por uma decisão da família.

O fato de muitos alunos mudarem de escola não implica, necessariamente, um aumento da segregação escolar, por exemplo, se as transferências forem aleatórias. No entanto, se pouquíssimos alunos trocarem de escola, seria possível antecipar que o impacto das transferências seria baixo ou nulo nos padrões de segregação. A Tabela 1 apresenta a proporção de alunos que trocaram de escola. Replicando as análises para a coorte de alunos do $1^{\circ}$ ano em 2007, é possível observar que os resultados são muito semelhantes (BARTHOLO, 2014).

TABELA 1 - Proporção de alunos no primeiro segmento do ensino fundamental que trocaram de escola - Coorte 2006, $1^{\circ}$ ano

Em porcentagem

\begin{tabular}{l|c|c|c|c}
\hline \multicolumn{1}{c|}{ TROCAS } & $\mathbf{2 0 0 6 - 2 0 0 7}$ & $\mathbf{2 0 0 6 - 2 0 0 8}$ & $\mathbf{2 0 0 6 - 2 0 0 9}$ & $\mathbf{2 0 0 6 - 2 0 1 0}$ \\
\hline Todas as trocas & 13,5 & 21,0 & 30,0 & 33,0 \\
\hline Trocas para uma escola próxima & 6,0 & 9,0 & 15,0 & 16,0 \\
\hline Fonte: Secretaria Municipal de Educação do Rio de Janeiro.
\end{tabular}

Há inúmeras razões para as famílias buscarem uma nova escola para seus filhos. Para os propósitos deste estudo, é interessante discriminar as transferências de escolas

4 Estamos aqui chamando de "questões pedagógicas" uma ampla gama de motivos que podem levar a família a efetuar a troca de escola, tais como a insatisfação da família com a escolha inicial (trabalho pedagógico deficiente, desorganização escolar, violência dentro ou ao redor da escola), ou ainda, quando a família é convencida pelo diretor da escola a trocar seu filho de escola. relacionadas a "questões pedagógicas"4 e aquelas ocorridas por outros motivos - por exemplo, mudança de endereço da família. É difícil fazer tal distinção, pois não há qualquer informação objetiva nas bases da SME-RJ sobre os motivos da transferência. Existem, no entanto, estudos qualitativos que apresentam dados sobre os critérios e estratégias utilizados pelas famílias para trocar seus filhos de escola na rede pública (COSTA; PRADO; ROSISTOLATO, 2013).

Além disso, há dois problemas com a informação sobre o endereço das famílias: dados faltantes; e o fato de que nem toda mudança de endereço implica, necessariamente, 
uma troca de escola (quando famílias se mudam para uma casa muito próxima da anterior, por exemplo). Diante desse desafio, a opção foi identificar apenas os alunos que foram transferidos para uma escola próxima da escolha anterior. ${ }^{5}$ A forma mais segura e simples de obter esse número é identificar os alunos que se mudaram para escolas que estão na mesma Coordenadoria Regional de Educação (CRE) e Polo. ${ }^{6}$

É provável que os valores referentes às trocas para uma escola próxima (Tabela 1) estejam subestimados, uma vez que escolas pertencentes a Polos diferentes podem situar-se geograficamente próximas. Trata-se, portanto, de uma estimativa conservadora do fenômeno de trocas de alunos entre as escolas.

É possível observar que um em cada três alunos mudou de escola ao longo das transições do primeiro segmento e pelo menos $16 \%$ foram transferidos para uma escola próxima da escolha inicial. Estamos lidando com um fenômeno pouquíssimo explorado nos estudos educacionais brasileiros. Há trabalhos nos EUA que observaram o fenômeno e tentaram medir seus impactos na trajetória escolar dos alunos (SAPORITO, 2003). Algumas questões devem ser colocadas: por que tantos alunos trocam de escola? O que motiva essas trocas e qual seu impacto na segregação escolar? E, possivelmente, a questão mais importante: há consequência para a trajetória escolar dos alunos que mudam de escola? Este estudo objetiva responder pelo menos uma dessas questões, ou seja, o impacto das transferências de alunos na segregação escolar.

O Gráfico 1 apresenta os valores do GS em um desenho transversal. Em função das altas taxas de repetência e do envio de alunos para as classes de realfabetização (ao final do $3^{\circ}$ ano em 2008), os valores observados devem ser interpretados com cuidado. ${ }^{7}$ Trata-se da primeira tentativa de captar o impacto das transferências dos alunos na segregação escolar. Aqui, os efeitos da reprovação e do envio de alunos para classe de realfabetização estão misturados com o potencial impacto das transferências de alunos.
5 Escola próxima significa uma distância que, em geral, se pode percorrer a pé.

6 Os Polos de matrícula eram utilizados anteriormente (antes da introdução da nova regra de admissão de alunos) para organizar os procedimentos de matrícula na rede. Os Polos apresentam uma área geográfica concisa, que em geral pode ser percorrida a pé.

7 Na coorte analisada, 14\% dos alunos ficaram reprovados na transição do 3ํ para o 4 ano e $6 \%$ foram enviados para as classes de realfabetização - ver Bartholo (2014). 
GRÁFICO 1 - Padrões de segregação escolar desenho transversal primeiro segmento do ensino fundamental (2006-2010)

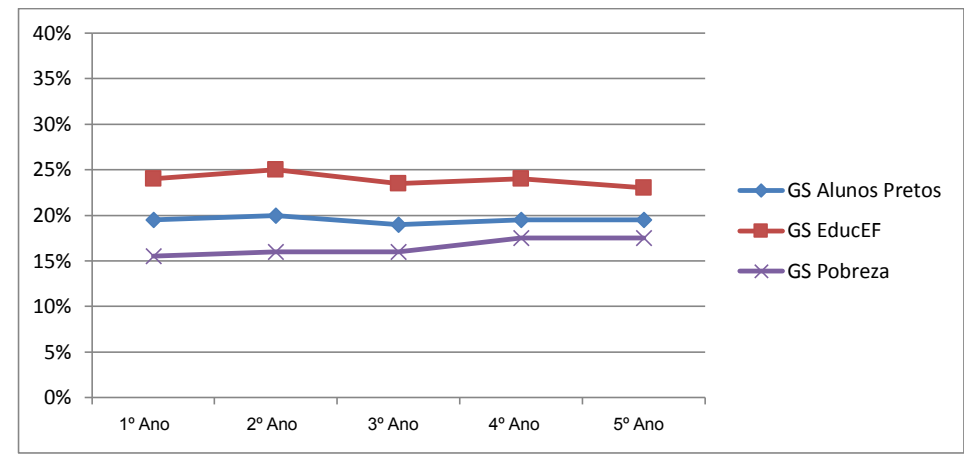

Fonte: Secretaria Municipal de Educação do Rio de Janeiro.

Os dados do Gráfico 1 não são conclusivos quando se observa o comportamento das três variáveis (educação dos pais foi calculada de duas formas distintas). É possível verificar grande estabilidade nas variáveis, com exceção de GS pobreza, que registra um pequeno aumento a partir da transição do $3^{\circ}$ para $o 4^{\circ}$ ano.

O Gráfico 2 traz os dados para o primeiro modelo longitudinal, considerando as transições do primeiro segmento (2006-2010). O novo modelo apresenta um resultado ligeiramente diferente do desenho transversal. Os cálculos do GS para as três variáveis sugerem um aumento pequeno e constante nos níveis gerais de segregação. É possível observar um aumento mais pronunciado na transição do $3^{\circ}$ para $04^{\circ}$ ano. A pergunta é: por que os dois modelos sugerem padrões levemente distintos? 
GRÁFICO 2 - Padrões de segregação escolar desenho longitudinal 1 - primeiro segmento do ensino fundamental (2006-2010)

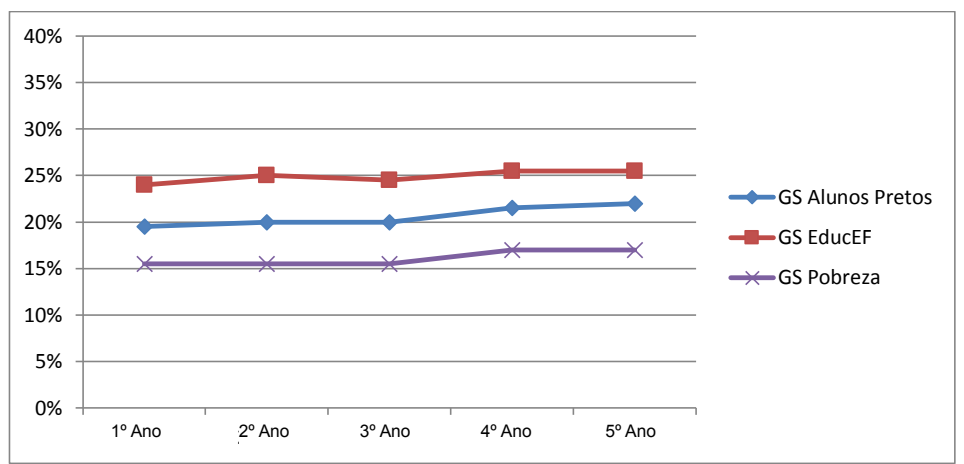

Fonte: Secretaria Municipal de Educação do Rio de Janeiro.

É razoável presumir que a mudança nos padrões observados no Gráfico 2 , a partir da transição do $3^{\circ}$ para o $4^{\circ}$ ano, esteja relacionada à concentração de reprovação de alunos no $3^{\circ}$ ano, bem como ao período de envio de alunos para a chamada classe de realfabetização. Bartholo (2014), analisando o perfil dos alunos e das escolas referente à reprovação e ao envio de alunos para classe de realfabetização, observou que os alunos em desvantagem potencial e/ou alocados em escolas de baixa performance têm mais chance de serem reprovados ou enviados para a classe de realfabetização. Tais evidências sugerem que parte do aumento verificado nos indicadores de segregação no Gráfico 2, provavelmente, não está relacionada unicamente com a transferência de alunos entre as escolas.

O último modelo também é longitudinal, mas com a diferença que os alunos reprovados ao longo das transições ou enviados para as classes de realfabetização não são excluídos das análises. A única informação considerada é a mudança de escola. O objetivo é tentar "isolar" o efeito das transferências de alunos na segregação escolar. 
GRÁFICO 3 - Padrões de segregação escolar desenho longitudinal 2 - primeiro segmento do ensino fundamental (2006-2010)

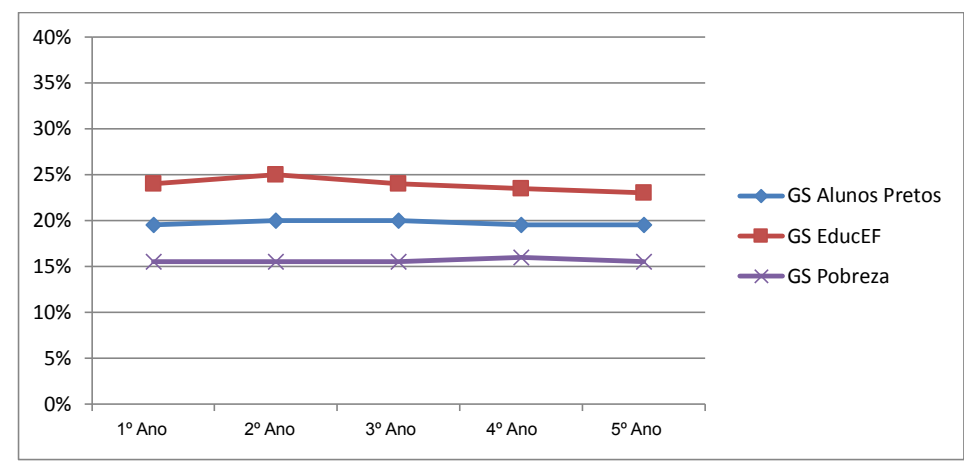

Fonte: Secretaria Municipal de Educação do Rio de Janeiro.

Mais uma vez, os padrões de segregação calculados sugerem estabilidade ao longo do tempo. Os três modelos apresentados para o primeiro segmento não corroboram a hipótese inicial. As transferências de alunos, apesar de intensas, não parecem apresentar um padrão específico que associa o perfil dos alunos à composição social das escolas. As evidências iniciais sugerem que as transferências de alunos não tornam as escolas do ensino fundamental inicial mais segregadas.

\section{SEGUNDO SEGMENTO DO ENSINO FUNDAMENTAL}

$\mathrm{O}$ segundo segmento apresenta um total de quatro séries e três transições. A hipótese inicial é igual àquela apresentada para o primeiro segmento e sugere que as trocas de alunos entre escolas têm um padrão específico, que associa o perfil dos alunos à composição social das escolas. As quatro séries finais do ensino fundamental, na rede municipal do Rio de Janeiro, englobam aproximadamente 450 escolas e $250 \mathrm{mil}$ alunos. A coorte analisada corresponde a 83555 alunos e há uma perda de casos (attrition rate) de $0,7 \%$ ao longo das três transições.

A Tabela 2 traz a proporção de alunos que mudaram de escola ao longo do segundo segmento. Os cálculos foram replicados para a coorte de alunos do $6^{\circ}$ ano do ensino fundamental de 2007 e os resultados são muito semelhantes. 
Observa-se que a proporção de alunos que efetivamente trocaram de escola no segundo segmento é menor do que aquela referente ao primeiro segmento.

TABELA 2 - Proporção de alunos no segundo segmento do ensino fundamental que trocaram de escola - coorte $2006,6 \circ$ ano

\begin{tabular}{l|c|c:c}
\multicolumn{1}{c}{ EROCAS } & $\mathbf{2 0 0 6 - 2 0 0 7}$ & $\mathbf{2 0 0 6 - 2 0 0 8}$ & $\mathbf{2 0 0 6 - 2 0 0 9}$ \\
\hline Todas as trocas & 10,0 & 16,0 & 18,0 \\
\hline Trocas para uma Escola próxima & 4,0 & 5,0 & 6,0 \\
\hline
\end{tabular}

Fonte: Secretaria Municipal de Educação do Rio de Janeiro.

Os números indicam que quase um quinto dos alunos mudou de escola ao longo do ensino fundamental e pelo menos $6 \%$ desses alunos optaram por uma nova escola próxima geograficamente da escolha inicial. Poder-se-ia especular que as transferências entre os alunos do segundo segmento seriam mais frequentes, se comparadas com as dos seus pares do primeiro segmento, uma vez que são alunos mais velhos e com maior autonomia para locomoção, usando o benefício do RioCard. ${ }^{8}$

Entretanto, os dados sugerem que a troca de escola é mais frequente entre os alunos mais novos. Uma possível explicação seria a maior oferta de escolas no ensino fundamental inicial, o que possibilitaria transferências mais frequentes. ${ }^{9}$ Estudos qualitativos devem investigar outras explicações para o fenômeno descrito.

O Gráfico 4 apresenta os valores do GS em um desenho transversal considerando os dados da coorte do segundo segmento do ensino fundamental. Os cálculos do primeiro modelo (transversal) devem ser interpretados com cuidado pelas mesmas razões explicitadas no primeiro segmento. Há dois fatores que podem interferir nas análises sobre as trocas de alunos entre escolas: repetência; e envio de alunos para programas que buscam corrigir a distorção idade-série.

No segundo segmento, há um pico de reprovação na transição entre o $6^{\circ}$ e $7^{\circ}$ ano (BARTHOLO, 2014). Além disso, é possível observar o envio de alunos para classes de progressão ou para o Programa de Educação de Jovens e Adultos (Peja II) ao longo do segundo segmento.
8 Os alunos recebem um cartão, o RioCard, que permite a utilização de transporte público (ônibus e metrô) sem que haja custo financeiro para as famílias (Projeto de emenda à Lei Orgânica do Município do Rio de Janeiro, n. 14/2010, Art. 401).

9 O número de escolas no ensino fundamental inicial é quase o dobro comparado com a etapa subsequente. 
GRÁFICO 4 - Padrões de segregação escolar desenho transversal - segundo segmento do ensino fundamental (2006-2009)

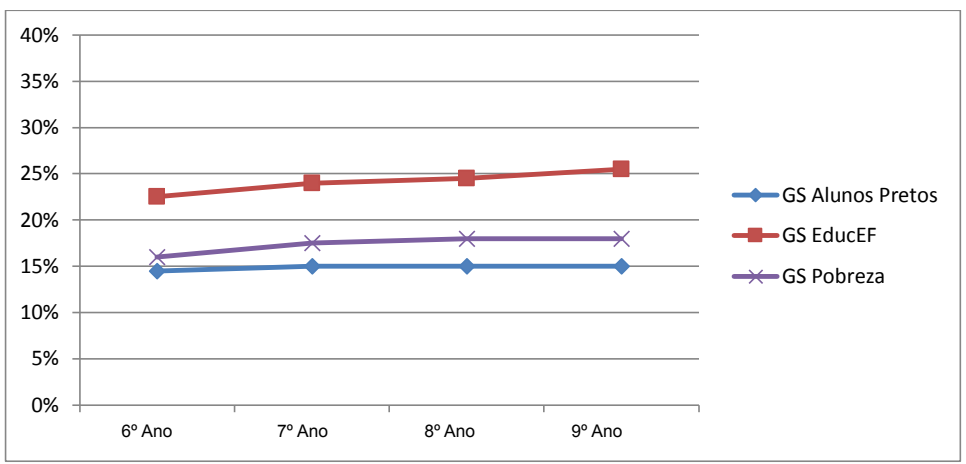

Fonte: Secretaria Municipal de Educação do Rio de Janeiro.

É possível observar um aumento pequeno e gradual em todos os cálculos do GS, com destaque para pobreza e pais que não completaram o ensino fundamental (EducEF). Os dados do modelo transversal corroboram a hipótese inicial, sugerindo que as escolas vão apresentando maior segregação ao longo das transições no segundo segmento. Os modelos longitudinais irão confirmar esse primeiro resultado?

O Gráfico 5 apresenta os dados do primeiro modelo longitudinal, considerando nas análises apenas os alunos que foram aprovados e permaneceram nas turmas regulares do segundo segmento do ensino fundamental. Os cálculos do GS mais uma vez corroboram a hipótese inicial e sugerem aumento constante e gradual em todas as variáveis.

GRÁFICO 5 - Padrões de segregação escolar desenho longitudinal 1 - segundo segmento do ensino fundamental (2006-2009)

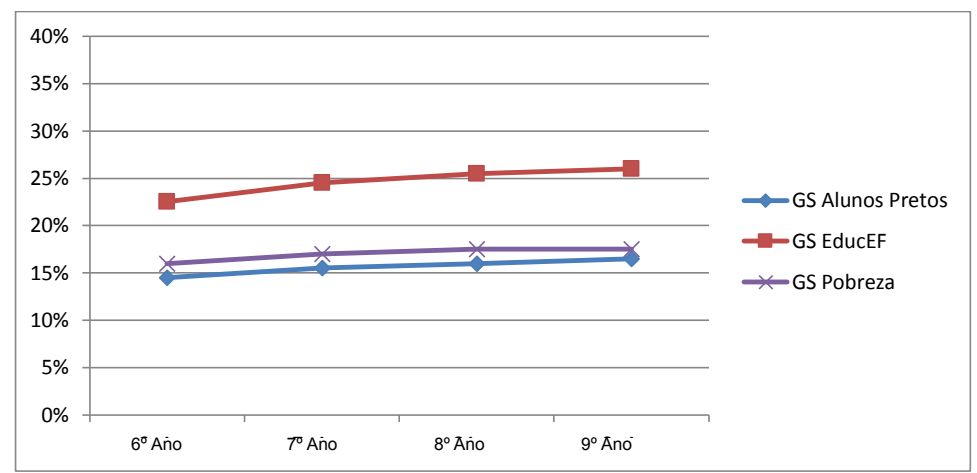

Fonte: Secretaria Municipal de Educação do Rio de Janeiro. 
Observa-se que a elevação das variáveis no Gráfico 5 é ligeiramente maior do que no Gráfico 4. Cabe ressaltar que a maioria das reprovações do segundo segmento ocorreu na transição do $6^{\circ}$ para o $7^{\circ}$ ano, porém os cálculos do GS sugerem um aumento constante ao longo de todas as transições. Isso pode ser um indício de que as transferências de alunos entre escolas, nessa etapa do ensino fundamental, apresentam um padrão específico, que associa o perfil dos alunos à composição social das escolas. Alunos em desvantagem potencial teriam maior chance de serem transferidos para escolas que já possuem alta concentração de alunos com a mesma característica.

O Gráfico 6 mostra os dados do segundo modelo longitudinal. Nesse caso, os alunos reprovados ou enviados para classes de progressão ou Peja II não são excluídos da análise. Os cálculos do GS sugerem um aumento dos níveis de segregação em duas variáveis: pobreza e pais que não completaram o ensino fundamental.

GRÁFICO 6 - Padrões de segregação escolar desenho longitudinal 2 - segundo segmento do ensino fundamental (2006-2009)

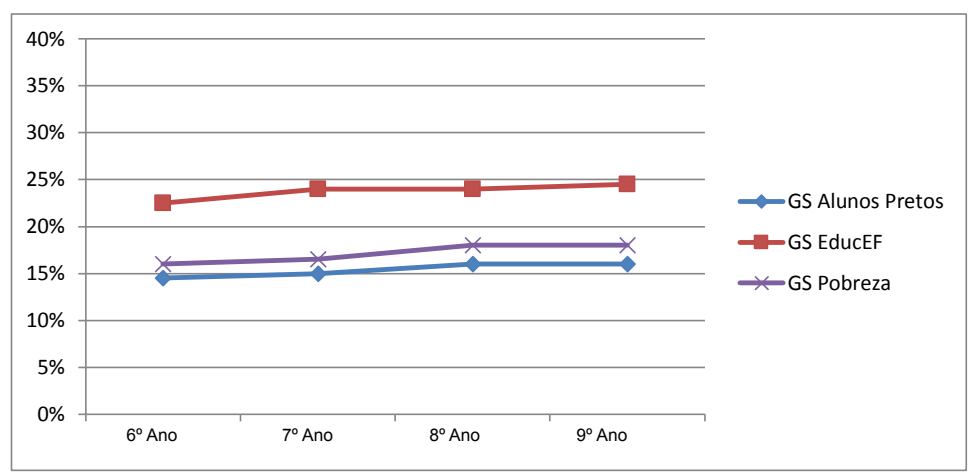

Fonte: Secretaria Municipal de Educação do Rio de Janeiro.

Esse último modelo (longitudinal 2) apresenta valores mais conservadores quando comparados aos Gráficos 4 e 5 . No entanto, é possível observar um padrão nos cálculos do GS, considerando os três modelos no segundo segmento do ensino fundamental. Ao longo das três transições do segundo segmento, é possível observar um aumento modesto, porém 
constante, dos valores calculados do GS. A consistência dos três modelos permite afirmar que as evidências iniciais para o segundo segmento corroboram a hipótese inicial do estudo.

\section{REMANEJAMENTO}

Esta seção analisa o impacto da transição entre o $5^{\circ}$ e o $6^{\circ}$ ano do ensino fundamental. Trata-se de uma transição ímpar, pois é um período obrigatório de troca de escolas para a maioria dos alunos (aproximadamente 90\% dos alunos). A hipótese inicial sugere um padrão nas transferências associando o perfil dos alunos à composição social das escolas. Haveria, portanto, um sistema de colaboração entre as escolas, o que permitiria a seleção de alunos pelas direções desses estabelecimentos (CARVALHO, 2014). Estudos anteriores concluíram que os chamados alunos em desvantagem potencial teriam menores chance de acesso às escolas de alta performance (BRUEL; BARTHOLO, 2012; COSTA et al., 2014).

A forma mais eficaz para observar o impacto do remanejamento na segregação escolar é comparar a alocação dos alunos antes e após a transição. Os modelos aqui utilizados são transversais e longitudinais (exatamente iguais àqueles anteriormente expostos) e serão replicados para mais de uma coorte para testar a consistência dos resultados. Os desenhos apresentados buscam responder a uma questão: qual o efeito geral do remanejamento de alunos entre o primeiro e o segundo segmentos na segregação escolar?

As tabelas 3 e 4 trazem os valores de GS em um modelo transversal para duas coortes distintas: 2008-2009; e 20092010. Se as indicações dos estudos anteriores estiverem corretas, os valores de GS calculados para o $6^{\circ}$ ano serão maiores (para todas as variáveis) se comparados com os valores iniciais do $5^{\circ}$ ano. 
TABELA 3 - Segregation Index - modelo transversal para o $5^{\circ}$ ano 2009 e $6^{\circ}$ ano 2010 do ensino fundamental

\begin{tabular}{l|c|c|c}
\multicolumn{1}{c|}{ VARIÁVEIS } & $\mathbf{5}^{\circ}$ ANO 2009 & 60 ANO 2010 & $\begin{array}{c}\text { Em porcentagem } \\
\text { PROPORCIÓNIO }\end{array}$ \\
\hline GS Alunos Pretos & 19,0 & 14,0 & 26,0 \\
\hline GS EducEF & 24,0 & 18,0 & 25,0 \\
\hline GS Pobreza & 19,0 & 16,5 & 13,0 \\
\hline
\end{tabular}

Fonte: Secretaria Municipal de Educação do Rio de Janeiro.

TABELA 4 - Segregation Index - modelo transversal para o $5^{\circ}$ ano 2008 e 6 ano 2009 do ensino fundamental

\begin{tabular}{l|c|c|c}
\multicolumn{1}{c|}{ VARIÁVEIS } & 50 ANO 2008 & 60 ANO 2009 & $\begin{array}{c}\text { Em porcentagem } \\
\text { PECLÍNIO } \\
\text { PROPORCIONAL }\end{array}$ \\
\hline GS Alunos Pretos & 18,0 & 14,0 & 22,0 \\
\hline GS EducEF & 24,0 & 19,0 & 21,0 \\
\hline GS Pobreza & 17,0 & 16,0 & 6,0 \\
\hline
\end{tabular}

Fonte: Secretaria Municipal de Educação do Rio de Janeiro.

Os valores em ambas as tabelas são muito semelhantes e refutam a hipótese inicial. Todas as variáveis sugerem uma diminuição da segregação escolar, com destaque especial para alunos negros e aqueles cujos pais não concluíram o ensino fundamental. O resultado é ainda mais surpreendente quando se observa o declínio proporcional relativo (diferença entre a segunda e a primeira medida, dividida pelo valor nominal da medida inicial). Trata-se de uma queda importante na segregação escolar, maior do que as mudanças verificadas nas transições ao longo do primeiro ou segundo segmento.

O Gráfico 7 ilustra a ruptura no padrão de segregação, medindo os níveis de segregação nas seis séries iniciais do ensino fundamental (2006-2011). 
GRÁFICO 7 - Padrões de segregação escolar desenho transversal - 1 até o 6ㅇ ano do ensino fundamental (2006-2011)

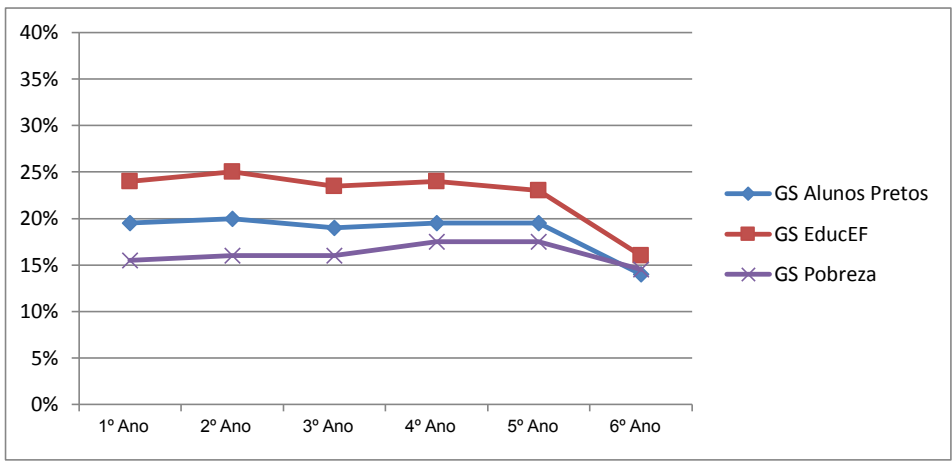

Fonte: Secretaria Municipal de Educação do Rio de Janeiro.

Como podemos interpretar esse resultado inesperado? Ainda mais importante, como explicar a aparente contradição entre os valores aqui apresentados e os achados de estudos anteriores? (BRUEL; BARTHOLO, 2012; COSTA et al., 2014). Os valores encontrados no modelo longitudinal são muito semelhantes aos exibidos nos modelos transversais e sugerem um declínio para todas as variáveis após o remanejamento.

TABELA 5 - Segregation Index - modelo longitudinal para o 5 ano 2009 e 6 ano 2010 do ensino fundamental

\begin{tabular}{l|c|c|c}
\multicolumn{1}{c|}{ VARIÁVEIS } & 50 ANO 2009 & 60 ANO 2010 & $\begin{array}{c}\text { Em porcentagem } \\
\text { DEOPOINIO } \\
\text { PROPCIONAL }\end{array}$ \\
\hline GS Alunos Pretos & $\mathbf{2 0 , 5}$ & 15,5 & 24,0 \\
\hline GS EducEF & 25,0 & 19,0 & 24,0 \\
\hline GS Pobreza & 20,0 & 16,5 & 17,0 \\
\hline
\end{tabular}

Fonte: Secretaria Municipal de Educação do Rio de Janeiro.

As limitações dos estudos anteriores que analisaram o remanejamento foram discutidas. $\mathrm{O}$ desenho apresentado nesse artigo tem a vantagem de utilizar dados para toda a população de alunos e mede a segregação escolar a partir de índices apropriados para análises longitudinais. Como interpretar o declínio na segregação escolar na transição do primeiro para o segundo segmento? Isso significa que as escolas estão intencionalmente alocando os alunos de 
uma forma mais equânime? A hipótese sobre uma possível seleção dos alunos estava errada? O mais provável é que a resposta para ambas as perguntas seja não.

A explicação mais plausível para o declínio na segregação não tem relação direta com a ação da burocracia educacional, mas sim com um elemento-chave que não foi observado nos estudos anteriores. Há uma redução importante de $45 \%$ no número de escolas na transição do primeiro para o segundo segmento. Isso aumenta as chances de as escolas ficarem com um perfil discente mais diversificado. Trata-se, portanto, de um efeito probabilístico, observado em outros sistemas educacionais quando ocorre uma diminuição importante no número de escolas.

Uma questão não respondida pelos modelos apresentados é se há indícios de seleção de alunos no remanejamento. Estudos futuros irão testar essa hipótese com a introdução de simulações que permitirão comparar os valores encontrados na distribuição real (apresentados pela primeira vez nesse artigo) contra uma distribuição verdadeiramente aleatória, que será obtida com a simulação da alocação dos alunos no remanejamento.

\section{CONCLUSÃO}

$\mathrm{O}$ artigo apresenta dados sobre um fenômeno pouco abordado no contexto brasileiro: a intensa troca de alunos entre escolas da mesma rede pública. Há poucos estudos que debatem o tema e buscam apresentar dados que permitam compreender as razões das mudanças e seus impactos na rede pública. A contribuição deste trabalho é descrever o fenômeno e medir seu impacto na segregação escolar.

Os dados indicam que um em cada três alunos matriculados no ensino fundamental trocou de escola e pelo menos $15 \%$ desses alunos (50 mil alunos considerando todas as matrículas no primeiro segmento) optaram por uma nova escola próxima da escolha inicial. As razões para o grande volume de transferências (em especial aquelas que ocorrem para escolas próximas geograficamente) devem ser investigadas em estudos futuros, no sentido de compreender as motivações e os mecanismos que as viabilizam. 
Ao contrário do que era previamente esperado, as trocas ao longo do segundo segmento foram menos frequentes, mas ainda muito constantes. Cerca de um quinto dos alunos mudou de escolas nas três transições do segundo segmento e pelo menos 6\% (15 mil alunos considerando todas as matrículas no segundo segmento) optaram por uma nova escola próxima da anterior. A principal explicação para a menor intensidade nas trocas é a menor oferta de escolas no segundo segmento. Há um decréscimo de $45 \%$ no total de escolas na transição do primeiro para o segundo segmento do ensino fundamental.

Ao todo foram testadas três hipóteses considerando três momentos distintos das transições ao longo do ensino fundamental. Os modelos apresentados sugerem que apenas nas transições do segundo segmento a hipótese foi corroborada. As evidências indicam que pelo menos parte do aumento observado na segregação escolar ao longo das transições no segundo segmento está relacionada às transferências de alunos entre as escolas. Aparentemente há um padrão nas trocas que associa o perfil dos alunos à composição social das escolas.

Os modelos para o primeiro segmento sugerem estabilidade ao longo dos anos, indicando que, apesar de intensas, as trocas de alunos não parecem aumentar a segregação escolar. Em outros termos, a despeito do grande volume de transferências, não há um padrão claro que associe o perfil dos alunos à composição social das escolas.

Por fim, os modelos para o remanejamento mostram um declínio dos níveis gerais de segregação. O resultado é o oposto do esperado e, em alguma medida, contradiz estudos anteriores que analisaram essa transição em especial. Os modelos aqui apresentados não permitem descartar a hipótese de uma seleção intencional de alunos no período do remanejamento. No entanto, é possível afirmar com segurança que o impacto geral do remanejamento não eleva a segregação, ao contrário, as escolas ficam com um perfil discente mais diversificado ou misturado.

A principal explicação para o quadro observado está relacionada à diminuição brusca no número de escolas. Trata-se, 
portanto, de um efeito probabilístico, já documentado em outros sistemas educacionais, quando ocorre uma redução relevante no total de escolas.

Todos os dados apresentados nesse estudo reforçam a importância de um debate sério, baseado em evidências, sobre as regras de acesso e permanência nas escolas. Há exemplos em diferentes países sobre sistemas educacionais que ampliaram o poder de escolha das famílias e criaram mecanismos formais para garantir que a opção das famílias seja respeitada. A experiência internacional indica claramente que as direções das escolas não devem ter poder de escolha sobre o seu alunado. Invariavelmente, quando isso ocorre, os atores selecionam seus alunos e amplificam as desigualdades educacionais (WEST; HIND; PENNELL, 2004; VAN ZANTEN, 2005).

As evidências iniciais apontadas nesse artigo podem servir de subsídio para estudos futuros que busquem compreender as causas das constantes trocas de escolas e também avancem no entendimento dos seus impactos na trajetória escolar dos alunos.

\section{REFERÊNCIAS}

ALVES, Maria Tereza G.; SOARES, José Francisco. O efeito das escolas no aprendizado dos alunos: um estudo com dados longitudinais com alunos do ensino fundamental. Educação e Pesquisa, São Paulo, v. 34, n. 3, p. 527-544, set./dez. 2008.

BARTHOLO, Tiago Lisboa. Measuring between-school segregation in an open enrolment system: the case of Rio de Janeiro. Journal of School Choice, n. 7, p. 353-371, 2013.

Segregação escolar na rede municipal do Rio de Janeiro: causas e consequências. 2014. Tese (Doutorado) - Programa de Pós-Graduação em Educação, Universidade Federal do Rio de Janeiro, Rio de Janeiro. 2014.

BRITO, Márcia de Souza T.; COSTA, Márcio da. Teachers’ practices and perceptions and their relations with prestige and school environment in public schools in the municipality of Rio de Janeiro. Revista Brasileira de Educação, Rio de Janeiro, v. 15, n. 45, p. 500-510, 2010.

BRUEL, Ana Lorena; BARTHOLO, Tiago Lisboa. Inequality of educational opportunities in Rio de Janeiro public school system: transition between segments of elementary school. Revista Brasileira de Educação, Rio de Janeiro, v. 17, n. 50, p. 303-328, 2012. 
CARVALHO, Julia Tavares. Segregação escolar e a burocracia educacional: uma análise da composição do alunado nas escolas municipais do Rio de Janeiro. 2014. Dissertação (Mestrado) - Programa de Pós-Graduação em Educação, Universidade Federal do Rio de Janeiro, Rio de Janeiro. 2014.

COSTA, Marcio; PRADO, Ana Pires; ROSISTOLATO, R. Talvez se eu tivesse algum conhecimento...: caminhos possíveis em um sistema educacional público e estratificado. Interseções, Rio de Janeiro, v. 14, p. 165-193, 2013.

COSTA, Marcio; KOSLINSKI, Mariane Campelo; BRUEL, Ana Lorena; BARTHOLO, Tiago de Souza. Movimentação de estudantes em um sistema educacional - padrões de "tracking", rotulagem e reprodução da estratificação social. Revista Universidade Federal de Juiz de Fora, 2014. No prelo.

DUNCAN, Otis Dudley; DUNCAN, Beverly. A methodological analyses of segregation indexes. American Sociological Review, v. 20, n. 2, p. 210-217, 1955.

EUROPEAN GROUP FOR RESEARCH ON EQUITY IN EDUCATIONAL SYSTEMS. Equity in European Educational Systems: a set of indicators. European Educational Research Journal, v. 4, n. 2, p. 1-151, 2005.

FITZ-GIBBON, Carol Taylor. Monitoring education: indicators, quality and effectiveness. London: Continuum, 1996.

GORARD, Stephen. Does the index of segregation matter? The composition of secondary schools in England since 1996. British Educational Research Journal, v. 35, n. 4, p. 639-652, 2009.

GORARD, Stephen; TAYLOR, Chris. A comparison of segregation indices in terms of strong and weak compositional invariance. Sociology, v. 36, n. 4, p. 875-895, 2002.

GORARD, Stephen; SEE, Beng Huat. Overcoming disadvantage in education. London: Routledge Falmer, 2013.

GORARD, Stephen; TAYLOR, Chris; FITZ, John. Schools, markets and choice policies. London: Routledge Falmer, 2003.

HAAHR, Jens Henrik; NIELSEN, Thomas Kibak; HANSEN, Martin Eggert; JAKOBSEN, Søren Teglgaard S. Explaining student performance: evidence from the international PISA, TIMSS and PIRLS surveys. Danish Technological Institute, 2005. Disponível em: <www.danishtechnology.dk>. Acesso em: 10 jan. 2012.

JENCKS, Christopher. Inequality: a reassessment of the effect of family and schooling in America. London: Lowe \& Brydone, 1972.

ROSENTHAL, Robert; JACOBSON, Lenore. Pygmalion in the classroom: teacher expectation and pupils' intellectual development. New York: Holst, Rinehart \& Winston, 1968.

SAPORITO, Salvatore. Private choices, public consequences: magnet school choice and segregation by race and poverty. Social Problems, v. 50, n. 2, p. 181-203, 2003. 
VAN ZANTEN, Agnès. Efeitos da concorrência sobre a atividade dos estabelecimentos escolares. Cadernos de Pesquisa, São Paulo, v. 35, n. 126, p. 565-593, 2005.

WEST, Anne; HIND, Audrey; PENNELL, Hazel. School admissions and "selection" in comprehensive schools: policy and practice. Oxford Review of Education, v. 30, n. 3, p. 347-369, 2004.

WILSON, William Julius. The truly disadvantaged: the inner city, the underclass and public policy. Chicago: University of Chicago Press, 1987.

YAIR, Gad. School organization and market ecology: a realist sociological look at the infrastructure of school choice. British Journal of Sociology of Education, v. 7, n. 4, p. 453-471, 1996.

\section{TIAGO LISBOA BARTHOLO}

Professor do Colégio de Aplicação da Universidade Federal do Rio de Janeiro (CAp/UFRJ). Diretor Adjunto de Licenciatura e Pesquisa do CAp/UFRJ. Bolsista da Coordenação de Aperfeiçoamento de Pessoal de Nível Superior (Capes) tiagobartholo@gmail.com 